Draft version August 25, 2021

Preprint typeset using IATEX style emulateapj v. 11/26/04

\title{
OUTLIERS FROM THE MASS-METALLICITY RELATION II: A SAMPLE OF MASSIVE METAL-POOR GALAXIES FROM SDSS
}

\author{
Molly S. Peeples, Richard W. Pogge, \& K. Z. Stanek \\ Department of Astronomy, Ohio State University, 140 W. 18th Ave., Columbus, OH 43210 , \\ molly@astronomy.ohio-state.edu, pogge@astronomy.ohio-state.edu, kstanek@astronomy.ohio-state.edu
}

(Dated: August 25, 2021)

Draft version August 25, 2021

\begin{abstract}
We present a sample of 42 high-mass low-metallicity outliers from the mass-metallicity relation of star-forming galaxies. These galaxies have stellar masses that $\operatorname{span} \log \left(M_{\star} / \mathrm{M}_{\odot}\right) \sim 9.4$ to 11.1 and are offset from the mass-metallicity relation by -0.3 to $-0.85 \mathrm{dex}$ in $12+\log (\mathrm{O} / \mathrm{H})$. In general, they are extremely blue, have high star formation rates for their masses, and are morphologically disturbed. Tidal interactions are expected to induce large-scale gas inflow to the galaxies' central regions, and we find that these galaxies' gas-phase oxygen abundances are consistent with large quantities of low-metallicity gas from large galactocentric radii diluting the central metal-rich gas. We conclude with implications for deducing gas-phase metallicities of individual galaxies based solely on their luminosities, specifically in the case of long gamma-ray burst host galaxies.

Subject headings: galaxies: abundances - galaxies: evolution
\end{abstract}

\section{INTRODUCTION}

Star-forming galaxies fall on a luminosity-metallicity relation such that more luminous galaxies tend to have higher gas-phase metallicities (the proxy for which is typically the oxygen abundance in units of $12+\log [\mathrm{O} / \mathrm{H}]$ ). This relation is observed to hold - shifted to lower metallicities - at redshifts as high as $z \sim 3.5$ (Erb et al. 2006; Maiolino et al. 2008), and its scatter decreases to $\sim 0.15$ dex at $z \sim 0$ when galaxy stellar mass replaces luminosity in the relation (Tremonti et al. 2004). Most commonly-accepted theories as to the origin of the mass-metallicity relation have as a central proposition that low-mass galaxies are metal-deficient, rather than that high-mass galaxies are metal-enhanced (Larson 1974; Dalcanton 2007; Finlator \& Davé 2008). In fact, there is some evidence that the mass-metallicity relation may flatten at large stellar masses, $\log \left(M_{\star} / \mathrm{M}_{\odot}\right) \gtrsim 10.5$ (e.g., Tremonti et al. 2004), though it is unclear to what extent this flattening is due to a saturation of the metallicity indicator used at high oxygen abundances (see e.g., Kewley \& Dopita 2002; Bresolin 2006; Kewley \& Ellison 2008).

Because most star-forming galaxies do lie on a massmetallicity locus, we can also learn about the gas-phase metallicity evolution of galaxies by studying the properties of galaxies that do not fall on the relation. In Peeples et al. (2008, hereafter Paper I), we explored the population of low-mass high-metallicity outliers, and postulated that these metal-rich dwarf galaxies have low gas fractions and are therefore nearing the end of substantial epochs of star formation. Here we investigate the other corner of the mass-metallicity plane by asking what we can learn about the evolution of massive galaxies from the properties of the high-mass low-metallicity outliers.

As shown in Figure 1, we find 42 low-metallicity highmass galaxies with masses ranging from $\log \left(M_{\star} / \mathrm{M}_{\odot}\right) \sim$ 9.4 to 11.1 and offsets from the central mass-metallicity relation of -0.3 to -0.85 dex. We describe in $\S 2$ how we selected this sample and verified the galaxies' outlier status. In $\S 3$, we describe the physical properties of these galaxies and discuss possible origins for their low oxygen abundances. Specifically, we find that they have highly disturbed morphologies strongly suggestive of merging or post-merging systems, are extremely blue, and have high specific star formation rates. We summarize our conclusions and state some implications of these findings in $\S 4$.

\section{SELECTING HIGH-MASS LOW-METALLICITY GALAXIES}

We began with a sample of $\sim 110000$ star-forming galaxies from the Sloan Digital Sky Survey (SDSS) Data Release 4 (Adelman-McCarthy et al. 2006) with gasphase abundances measured by Tremonti et al. (2004) and stellar masses derived using the techniques of Kauffmann et al. (2003). High-luminosity outliers from the mass-metallicity relation can be found in the lowmetallicity region of parameter space for one of two reasons: either they have spuriously low derived metallicities or they are genuine outliers. (The third possibility of an object having a spuriously high measured luminosity is much less likely.) In general, the strengths of the visible-wavelength oxygen forbidden [ $\left[\begin{array}{ll}\mathrm{O} & \mathrm{II}\end{array}\right]$ and [O III] emission lines increase as the oxygen abundance decreases; hence, it is possible for a bright galaxy with a weak active galactic nucleus (AGN) component to appear to have a low oxygen abundance due to contribution from the AGN itself. Likewise, an otherwise "red and dead" galaxy with strong [O II] emission might be labeled as star-forming and, in the Bayesian analysis of Tremonti et al., be assigned an artificially low metallicity. We therefore selected our sample of massive lowmetallicity galaxies by first ensuring that they are outliers in the luminosity- and mass-metallicity parameter spaces and then following up with several line-ratio diagnostic tests in an attempt to guarantee that their oxygen abundances relative to the rest of the sample are indeed believable. Images of the galaxies in our final sample are shown in Figure 2 and summary information is provided 


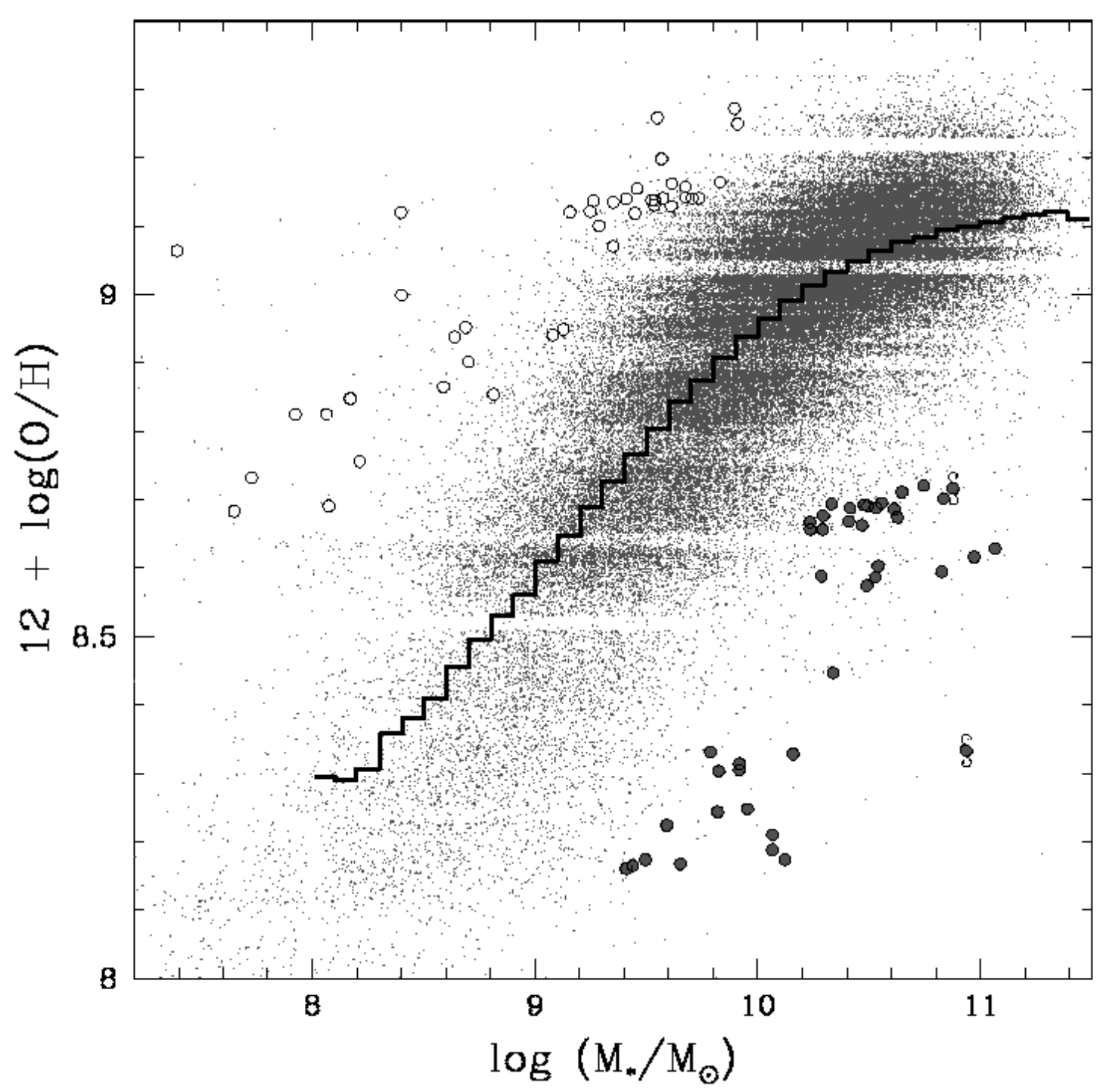

FIG. 1.- Plot of $12+\log (\mathrm{O} / \mathrm{H}) \mathrm{v} \cdot \log \left(\mathrm{M}_{\star} / \mathrm{M}_{\odot}\right)$. The small grey points represent galaxies from the main Tremonti et al. (2004) SDSS sample described in $\S 2$, and the step-curve is the median of these points in bins of 0.1 dex of total stellar mass. The high-mass low-metallicity massive galaxies in our sample are plotted as the large grey circles in the lower-right region of the diagram; the only two clearly spiral galaxies in the sample are denoted with " $\S$ " symbols. For reference we also plot the high-metallicity outliers from Paper I as large open points (upper left).

in Table 1. As only two of the galaxies in Figure 2 are clearly undisturbed spirals, we note these two galaxies in Table 1, Figure 1, and subsequent figures with "§" symbols.

\subsection{Sample Selection}

We summarize the selection process for our massive metal-poor galaxy sample in Table 2 . We begin by defining the parent galaxy sample from which our galaxies are outliers. Starting with the entire Tremonti et al. (2004) sample of star-forming galaxies, we choose only those galaxies with SDSS magnitude errors in the $g, r, i$, and $z$-bands of $<0.1 \mathrm{mag}$. In the $u$-band we take those galaxies that satisfy the conditions

$$
\begin{gathered}
\sigma(u)<0.1 \text { for } u<18 \\
\sigma(u)<\left(\frac{0.1}{1.5}\right) u+16.5 \text { for } 18 \leq u<19.5, \text { and }
\end{gathered}
$$

$$
\sigma(u)<0.2 \text { for } u \geq 19.5
$$

where $u$ is the observed $u$-band magnitude and $\sigma(u)$ is the SDSS error on $u$. These cuts yield a parent population of 86754 galaxies. To find the low-metallicity outliers from the luminosity-metallicity locus, we take bins of absolute $g$-band magnitude $M_{g}$ of width $0.4 \mathrm{mag}$, and each bin we take the $1 \%$ of objects with the smallest $12+\log (\mathrm{O} / \mathrm{H})$, as demonstrated in Figure 3. Because we do not want to preferentially select blue objects, we similarly take the $1 \%$ of galaxies with the smallest $12+\log (\mathrm{O} / \mathrm{H})$ in bins of $M_{z}$ of width $0.4 \mathrm{mag}$. Finally, we ensure that these galaxies are also outliers in the mass-metallicity plane by taking the $2.5 \%$ smallest $12+\log (\mathrm{O} / \mathrm{H})$ galaxies in bins of stellar mass of width $\Delta \log \mathrm{M}_{\star}=0.1 \mathrm{dex}$ and the $2.5 \%$ largest $\log \mathrm{M}_{\star}$ galaxies in bins of $12+\log (\mathrm{O} / \mathrm{H})$ of width 0.1 dex. These selection criteria yield a sample of 113 candidates for detailed evaluation and further downselection of the true low-metallicity outliers. 

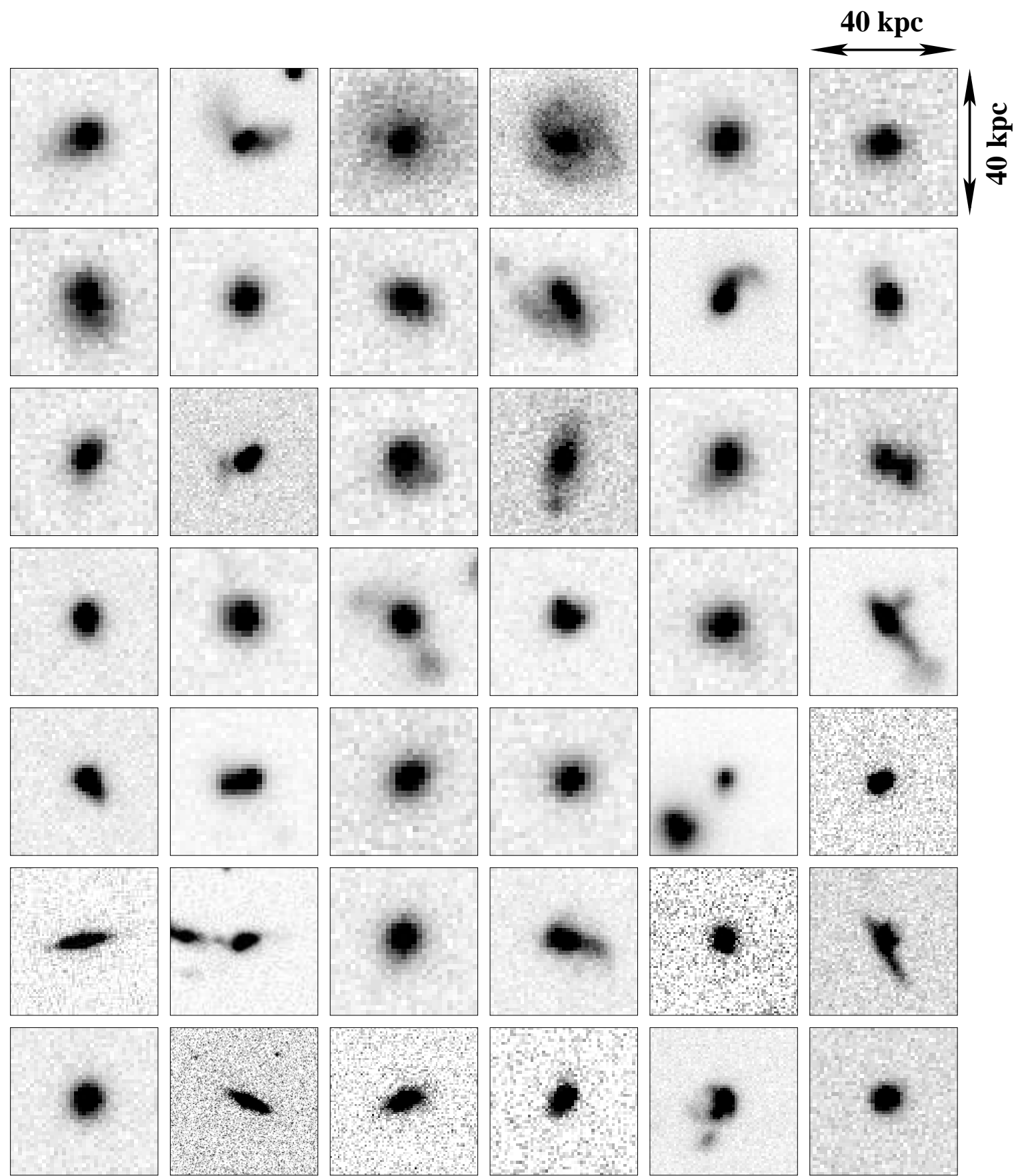

FIG. 2.- Normalized SDSS $g$-band images of massive low-metallicity galaxies scaled to $40 \times 40 \mathrm{kpc}$, with mass decreasing to the right and down from $\log M_{\star} \approx 11.1$ to 9.4 . The only two clearly spiral galaxies are the middle two on the top row.

One possible contamination source of these seemingly bright metal-poor galaxies is a low-level AGN, such as a low-ionization nuclear emission-line region (LINER). While it is possible for galaxies with weak AGN activity to also have low oxygen abundances, we choose to not try to disentangle these two effects on the emission line fluxes. Using the line fluxes measured by Tremonti et al. $(2004)^{1}$, we put these galaxies on the standard Baldwin, Phillips, \& Terlevich (BPT) 1981 diagrams. In particular, as shown in Figure 4, we find that about half of

\footnotetext{
${ }^{1}$ See http://www.mpa-garching.mpg.de/SDSS/DR4/raw_data.html for this very rich dataset.
}

these outliers have strong enough $[\mathrm{O} \mathrm{I}] \lambda 6300$ to place them in the "AGN" region of the BPT diagram. (We note that while the Tremonti et al. (2004) galaxies were chosen to be star-forming based on where they fall in the $[\mathrm{O} \mathrm{III}] / \mathrm{H} \beta$ v. $[\mathrm{N} \mathrm{II}] / \mathrm{H} \alpha$ plane, $3.4 \%$ of the parent sample galaxies fall in the $\mathrm{AGN}$ region of the $[\mathrm{O} \mathrm{III}] / \mathrm{H} \beta \mathrm{v}$. $[\mathrm{O} \mathrm{I}] / \mathrm{H} \alpha$ plane.) As expected, the strong-[O I] galaxies also fall close to the "composite" H II-AGN boundary as defined by Kewley et al. (2006b) in the $[\mathrm{O} \mathrm{III]} / \mathrm{H} \beta$ v. $[\mathrm{N} \mathrm{II}] / \mathrm{H} \alpha$ plane. Visual inspection of the spectra and images of these bogus outliers reveals that many of them are quite red, with only strong [O II] $\lambda 3727$ and perhaps weak $\mathrm{H} \alpha$ emission being suggestive of ongoing star formation. Because $[\mathrm{O} \mathrm{I}] \lambda 6300$ is a relatively weak line and 
TABLE 1

LOW-METALLICITY MASS-METALLICITY OUTLIERS

\begin{tabular}{|c|c|c|c|c|}
\hline $\mathrm{RA}$ & dec & $12+\log (\mathrm{O} / \mathrm{H})$ & $\log \mathrm{M}_{\star}$ & redshift \\
\hline 149.9796 & 10.24727 & 8.63 & 11.07 & 0.2423 \\
\hline 255.5060 & 60.79618 & 8.62 & 10.97 & 0.1257 \\
\hline 191.3246 & 4.87648 & 8.33 & 10.94 & $0.1800^{\S}$ \\
\hline 168.6324 & 48.91903 & 8.72 & 10.88 & $0.1116^{\S}$ \\
\hline 197.4139 & 62.76859 & 8.70 & 10.84 & 0.2587 \\
\hline 329.9707 & 1.03852 & 8.59 & 10.83 & 0.2200 \\
\hline 150.2157 & 8.30726 & 8.72 & 10.75 & 0.2290 \\
\hline 148.0473 & 54.31252 & 8.71 & 10.65 & 0.2544 \\
\hline 146.8469 & 53.06987 & 8.67 & 10.63 & 0.2471 \\
\hline 214.2315 & 40.44983 & 8.69 & 10.61 & 0.2060 \\
\hline 196.1901 & 62.40580 & 8.70 & 10.56 & 0.1118 \\
\hline 206.4954 & 11.47993 & 8.60 & 10.54 & 0.2373 \\
\hline 128.9575 & 44.87386 & 8.69 & 10.53 & 0.2264 \\
\hline 158.5174 & 6.20286 & 8.59 & 10.53 & 0.1043 \\
\hline 140.1770 & 0.84295 & 8.69 & 10.49 & 0.2521 \\
\hline 118.9094 & 33.44449 & 8.57 & 10.49 & 0.1413 \\
\hline 170.4012 & 0.54706 & 8.69 & 10.48 & 0.2292 \\
\hline 17.1399 & -0.46871 & 8.66 & 10.47 & 0.2270 \\
\hline 158.5274 & 5.51848 & 8.69 & 10.41 & 0.1708 \\
\hline 181.9754 & 12.39080 & 8.67 & 10.41 & 0.2613 \\
\hline 200.4299 & 43.40588 & 8.45 & 10.34 & 0.1950 \\
\hline 126.2741 & 7.21643 & 8.69 & 10.33 & 0.1974 \\
\hline 349.5542 & -0.69060 & 8.68 & 10.29 & 0.2517 \\
\hline 218.7960 & 44.18318 & 8.66 & 10.29 & 0.1276 \\
\hline 250.6480 & 42.39715 & 8.59 & 10.29 & 0.1511 \\
\hline 244.4054 & 35.82085 & 8.66 & 10.24 & 0.2259 \\
\hline 196.2852 & 53.19373 & 8.67 & 10.23 & 0.2719 \\
\hline 146.2970 & 42.64472 & 8.33 & 10.16 & 0.2576 \\
\hline 240.8967 & 31.83226 & 8.17 & 10.12 & 0.1544 \\
\hline 127.9271 & 51.42651 & 8.21 & 10.07 & 0.0813 \\
\hline 153.3421 & 2.58265 & 8.19 & 10.07 & 0.0780 \\
\hline 48.8150 & -7.76643 & 8.25 & 9.95 & 0.0612 \\
\hline 129.3898 & 47.96454 & 8.31 & 9.92 & 0.2152 \\
\hline 234.2552 & 31.44251 & 8.30 & 9.92 & 0.1538 \\
\hline 233.5084 & -1.96201 & 8.30 & 9.83 & 0.0787 \\
\hline 1.2987 & 1.06057 & 8.24 & 9.82 & 0.1028 \\
\hline 156.5582 & 48.74970 & 8.33 & 9.79 & 0.1604 \\
\hline 226.0778 & 2.76445 & 8.17 & 9.65 & 0.0350 \\
\hline 345.7720 & 1.24968 & 8.22 & 9.59 & 0.0690 \\
\hline 168.1920 & 1.33819 & 8.17 & 9.49 & 0.1088 \\
\hline 219.6623 & 53.14538 & 8.16 & 9.44 & 0.0900 \\
\hline 197.1532 & 59.88513 & 8.16 & 9.41 & 0.1410 \\
\hline
\end{tabular}

Note. - Sample of massive low-metallicity galaxies, reverse sorted by stellar mass. RA and Dec are in degrees (J2000.0), $12+\log (\mathrm{O} / \mathrm{H})$ and stellar masses are from Tremonti et al. (2004).

$\S$ Spiral galaxy.

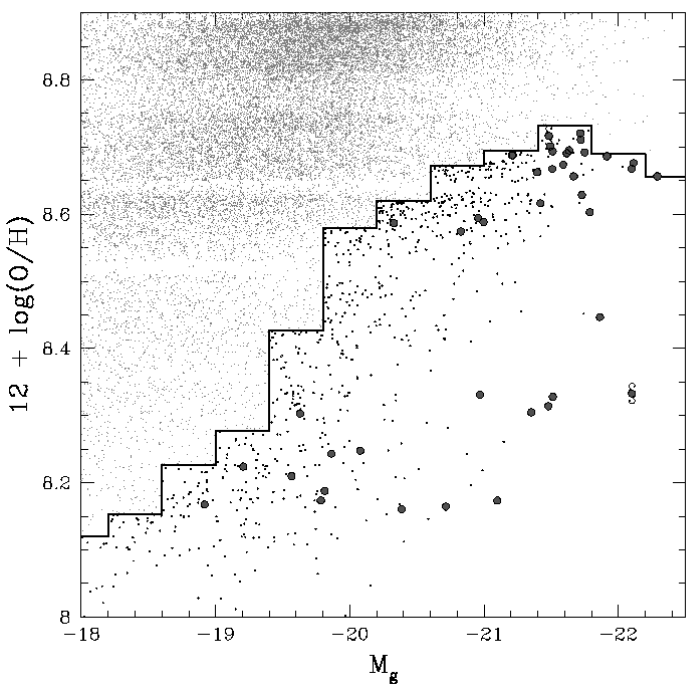

FIG. 3.- An example of one of the cuts made to find lowmetallicity high-luminosity outliers from the mass-metallicity relation. Plotted is $12+\log (\mathrm{O} / \mathrm{H})$ vs. $M_{g}$ for the main sample (small light grey points), the $1 \%$ objects the lowest $\log (\mathrm{O} / \mathrm{H})$ in bins of $M_{g}$ of width $0.4 \mathrm{mag}$ (small black points), and the final sample of high-mass low-metallicity outliers (large grey points). The only two clearly spiral galaxies in the sample are denoted with " $\S$ " symbols. The histogram shows the $1 \%$ threshold in $12+\log (\mathrm{O} / \mathrm{H})$ in bins of $M_{g}$.

many of the outliers are clustered near the the Kewley et al. (2006b) boundary, we kept galaxies whose $[\mathrm{O} \mathrm{I}] / \mathrm{H} \alpha$ ratio is within $2-\sigma$ of the $\mathrm{H}$ II-AGN boundary and whose spectra passed a visual inspection test for, e.g., clear $\mathrm{H} \beta$ emission. Representative spectra are shown in Figure 5.

We further tested whether or not the measured metallicities could be due to measurement errors in the relevant line fluxes. Using the Data Release 6 SDSS spectra (Adelman-McCarthy et al. 2008), we subtracted the underlying stellar continuum using the STARLIGHT program (Cid Fernandes et al. 2005), which simultaneously corrects for reddening and extinction using the Cardelli et al. (1989) extinction curves. While the extinction corrections are all small $\left(A_{V}<1\right)$, the Tremonti et al. line fluxes do not account for reddening, and thus a comparison of widely spaced line ratios produces a systematic offset. We therefore reject seven galaxies for which our measurements strongly disagree with Tremonti et al.'s on the $[\mathrm{N} \mathrm{II}] / \mathrm{H} \alpha$ ratio; the difference in each of these cases is such that using the Tremonti et al. ratio would result in a lower $12+\log (\mathrm{O} / \mathrm{H})$ than if ours were used. The added advantage to using the $[\mathrm{N} \mathrm{II}] / \mathrm{H} \alpha$ ratio is that this is the ratio used in the Pettini \& Pagel (2004) metallicity determination, which we use as discussed below.

\subsection{Metallicity Believability}

We note that we do not impose a metallicity error cut when selecting these low-metallicity outliers. This is because the errors on Tremonti et al.'s $12+\log (\mathrm{O} / \mathrm{H})$ are not a simple function of metallicity in this range. It has long been recognized that accurately measuring low oxygen abundances (e.g., using $R_{23}$ ) can be tricky due to degeneracies in the ionization parameter, and the line ratios usually used to determine the ionization parameter (e.g., [O III $] \lambda 5007 /[\mathrm{O}$ II $] \lambda 3727$ ) are also dependent on metallicity (McGaugh 1991; Kewley \& Dopita 2002). 


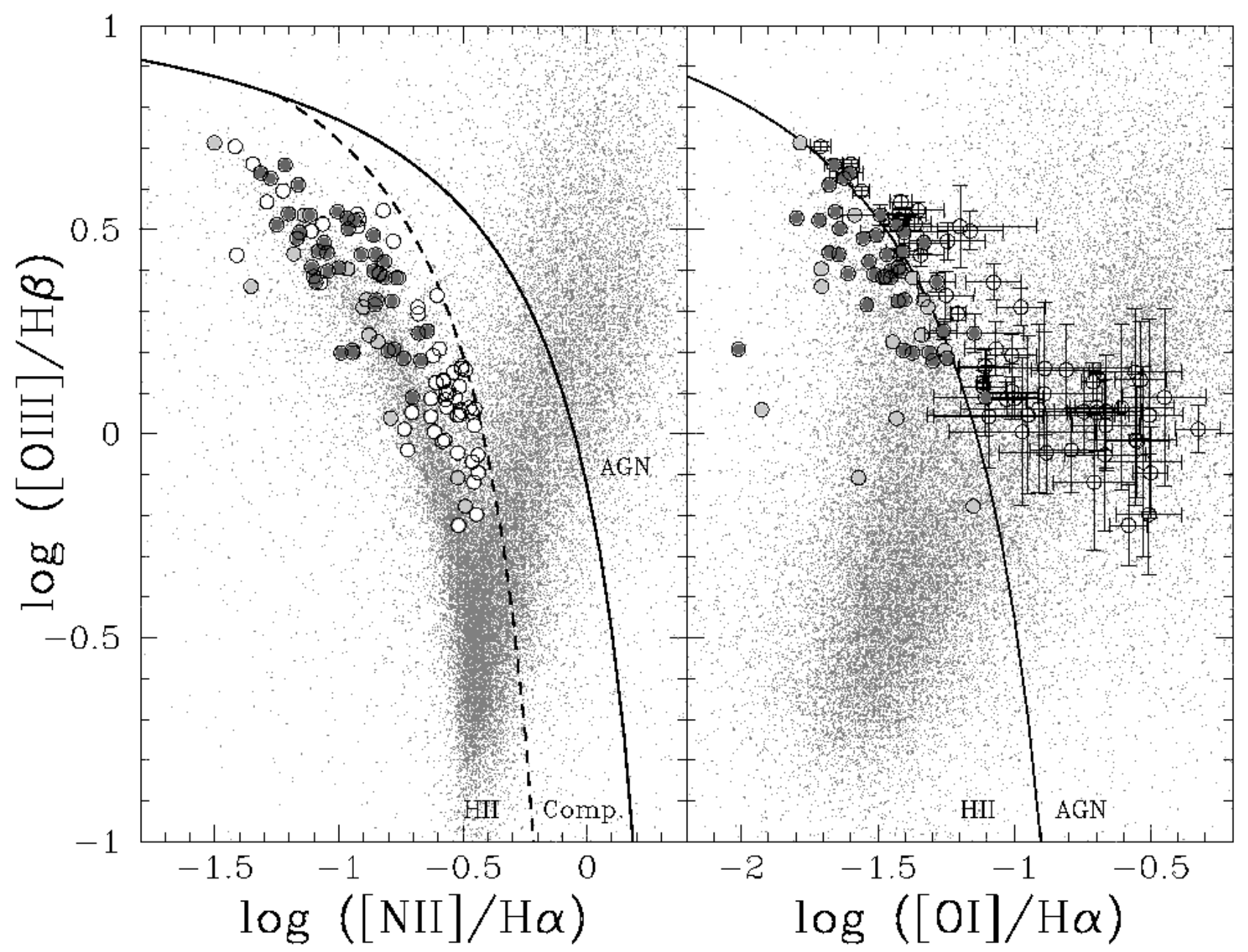

FIG. 4.- Baldwin, Phillips, \& Terlevich diagrams of $\log ([\mathrm{O} \mathrm{III}] / \mathrm{H} \beta)$ v. $\log ([\mathrm{N} \mathrm{II}] / \mathrm{H} \alpha)[$ left $] \operatorname{and} \log ([\mathrm{O} \mathrm{III}] / \mathrm{H} \beta)$ v. $\log ([\mathrm{O} \mathrm{I}] / \mathrm{H} \alpha)[$ right $]$. Plotted are the spectroscopic galaxies from SDSS DR4 (small grey points), the galaxies passing our luminosity, mass, and log $(\mathrm{O} / \mathrm{H})$ cuts but not in the final sample (open points), the galaxies passing the BPT diagram cuts (light grey points), and the galaxies in our final low-metallicity sample (dark grey points). Lines are taken from Kewley et al. (2006b).

Unfortunately, this means that many metallicity indicators are discontinuous or systematically tend to avoid particular abundance solutions.

We therefore adapted a different approach to ensure that our low-metallicity high-mass outliers geniunely have relatively low oxygen abundances. As discussed in $\S 2$, we first chose only those galaxies that lie in the $\mathrm{H}$ II region locus of the BPT diagrams, and secondly, we discarded those galaxies for which we did not measure a comparable $[\mathrm{N}$ II $] / \mathrm{H} \alpha$ ratio to those given by Tremonti et al.. Finally, we argue that if these galaxies are truly high-mass low-metallicity outliers, then they should be outliers regardless of how $12+\log (\mathrm{O} / \mathrm{H})$ is measured. (See Kewley \& Dopita 2002 and Kewley \& Ellison 2008 for thorough discussions of how and why different metallicity callibrations differ.) We therefore used the Tremonti et al. measurements recalculate $12+\log (\mathrm{O} / \mathrm{H})$ for the entire parent sample using the Pettini \& Pagel (2004) method, where the oxygen abundance is given by $12+\log (\mathrm{O} / \mathrm{H})=9.37+2.03 \times \mathrm{N} 2+1.26 \times \mathrm{N} 2^{2}+0.32 \times \mathrm{N} 2^{3}$,

where $\mathrm{N} 2 \equiv \log ([\mathrm{N} \mathrm{II}] \lambda 6584 / \mathrm{H} \alpha)$. The main disadvantage of this method is that it uses nitrogen as a proxy for oxygen; however, there are several factors that make it advantageous in these objects. First, it is reddening insensitive because the $[\mathrm{N} \mathrm{II}] \lambda 6584$ line and $\mathrm{H} \alpha$ are separated by only $\sim 20 \AA$. This means that we can safely use the Tremonti et al. measurements and our own measurements without being overly concerned about the reddening corrections. Second, unlike the various of $\mathrm{R}_{23}$ methods, the Pettini \& Pagel method is both continuous and single-valued throughout the metallicity range of interest; there are no "upper-" and "lower"-branches to concern us. We show the mass-metallicity relation with $12+\log (\mathrm{O} / \mathrm{H})$ measured with the Pettini \& Pagel (2004) method in Figure 6. Only one galaxy in the low-[O I] sample did not pass the $12+\log (\mathrm{O} / \mathrm{H})-\log \mathrm{M}_{\star}$ cuts described in Table 2 , suggesting that the remaining galaxies are true low-metallicity outliers.

We have made several other checks for potential systematics. First, spiral (and thus many star-forming) galaxies are known to have metallicity gradients such that their centers have higher abundances than at larger radii (e.g., Zaritsky et al. 1994; Kennicutt et al. 2003). It is therefore reasonable to expect that, on average, spiral galaxies with larger fractions of their light falling in the $3^{\prime \prime}$ SDSS fiber will have lower integrated metallicities. However, most of our galaxies (see Fig. 2) are not spiral 


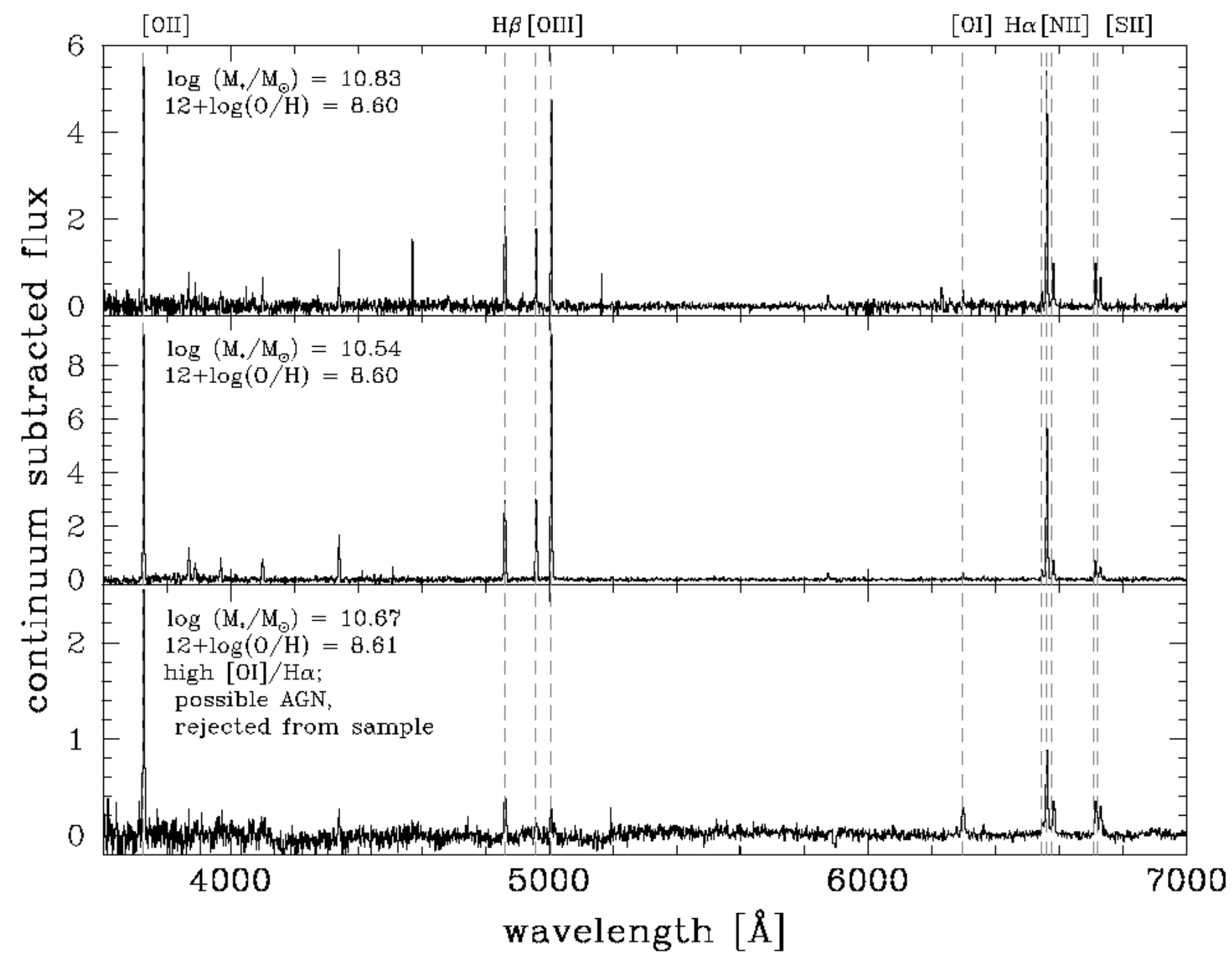

FIG. 5.- Sample spectra. Two low-metallicity high-mass outliers (top and middle panels) and one galaxy rejected as a potential AGN (bottom).

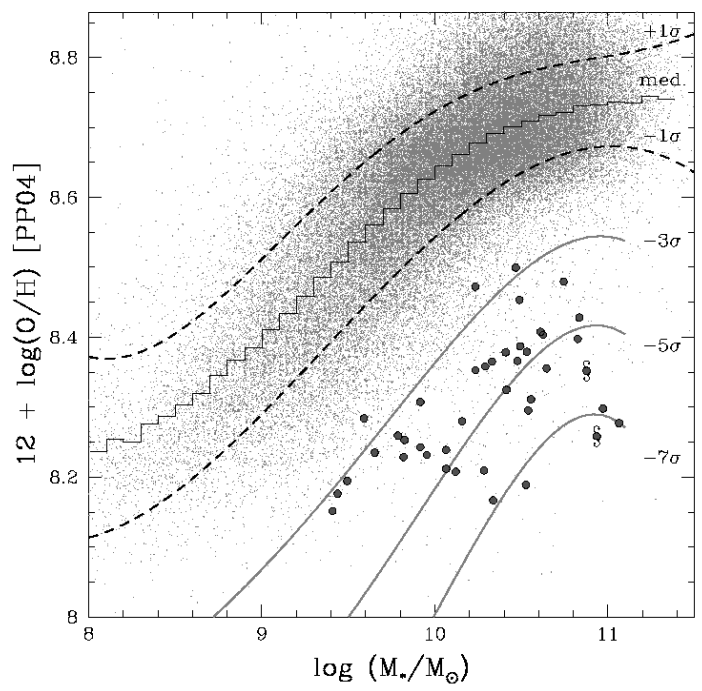

Fig. 6.- Plot of Pettini \& Pagel (2004) $12+\log (\mathrm{O} / \mathrm{H})$ v. $\log \left(\mathrm{M}_{\star} / \mathrm{M}_{\odot}\right)$. The galaxies in our sample are plotted as the large grey circles in the lower-right region of the mass-metallicity diagram; the only two clearly spiral galaxies in the sample are denoted with " $\S$ " symbols. The histogram denotes the median $12+\log (\mathrm{O} / \mathrm{H})$ in bins of stellar mass, while the curves are fits to standard deviation offsets as labeled. galaxies, so we do not expect for this to be a huge effect. Regardless, as shown in Figure 7, while the galaxies in our sample do have somewhat high fiber fractions, this is only a small effect, and the offsets we see in metallicity are much greater than can be explained by large fiber fractions, which would cause contamination of central high-metallicity H II regions by low-metallicity H II gas at larger galactocentric radii.

Similarly, the final redshift distribution for the galaxies in our sample peaks at higher $z$ than for the main sample; since we have convinced ourselves that this is not purely a fiber fraction effect, it is worrisome that it might partially be a redshift-evolution effect. When the universe was younger, galaxies are expected to have had lower oxygen abundances; the observed mass-metallicity relations for redshifts $z>0.1$ are systematically shifted to lower metallicity from what is observed for nearby galaxies (Erb et al. 2006; Maiolino et al. 2008). We plot our low-metallicity outliers against the observed massmetallicity evolution from Maiolino et al. (2008) in Figure 8. While the median redshift for galaxies in our sample is $z \sim 0.17$, the observed oxygen abundances are more typical of redshifts $2.2 \lesssim z \lesssim 3.5$. Though a large amount of scatter in the mass-metallicity relation is both expected and observed (Savaglio et al. 2005; Erb et al. 2006; 


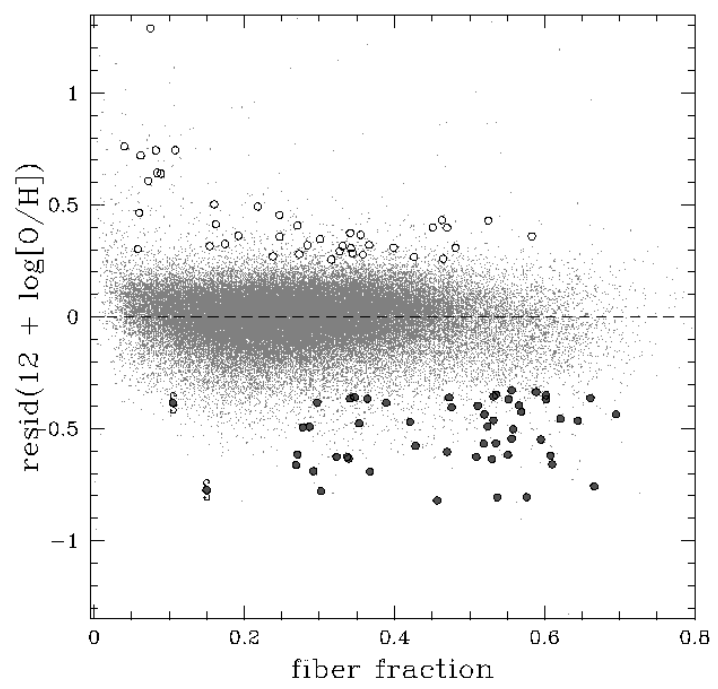

Fig. 7.- Plot of residual in $12+\log (\mathrm{O} / \mathrm{H})$ from the Tremonti et al. fit to the mass-metallicity relation v. fiber fraction. The high-mass low-metallicity outliers are shown as the large dark grey points at the bottom of the diagram, with the only two distinctly spiral galaxies in the sample as " $\S$ " symbols. For reference we plot the high-metallicity outliers from Paper I as large open points (top).

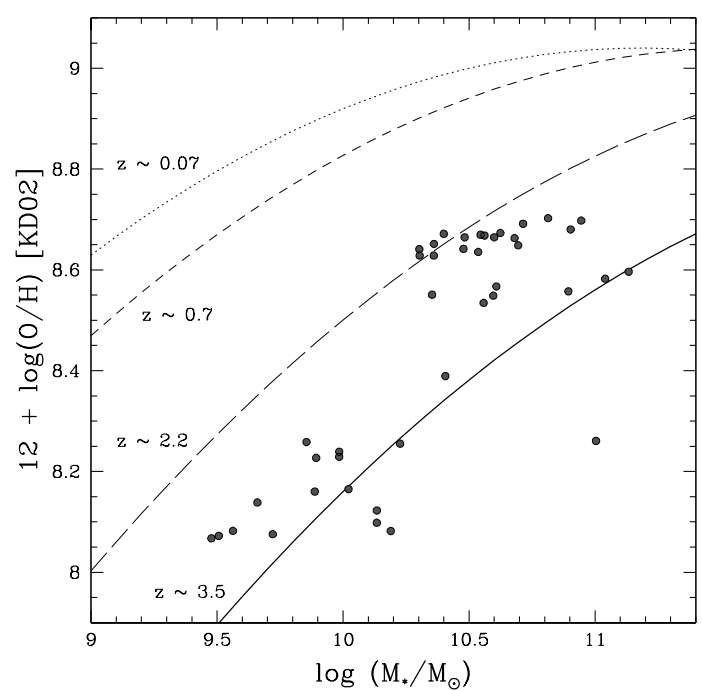

FIG. 8.- Evolution of the mass-metallicity relation from Maiolino et al. (2008) with comparison to low-metallicity highmass outliers (grey points). Fits to the mass-metallicity relation from Maiolino et al. (2008) are shown with lines: $z \sim 0.7$, dotted; $z \sim 0.7$, short dashed; $z \sim 2.2$, long dashed; and $z \sim 3.5$ solid. The grey points denote our high-mass low-metallicity outliers $(0.035<z<0.27)$, with re-calculated metallicities using the Kewley \& Dopita (2002) diagnostic using our measurements of the line ratios so that they are on the same scale as the Maiolino et al. results. (The stellar masses have also been shifted to match the Maiolino et al. fits.)

Kobayashi et al. 2007; Finlator \& Davé 2008; Maiolino et al. 2008) at all redshifts, the abundance offsets of our low-metallicity outliers are much more pronounced than the relatively subtle expectations from metallicity evolution with cosmic time.

\section{DISCUSSION}

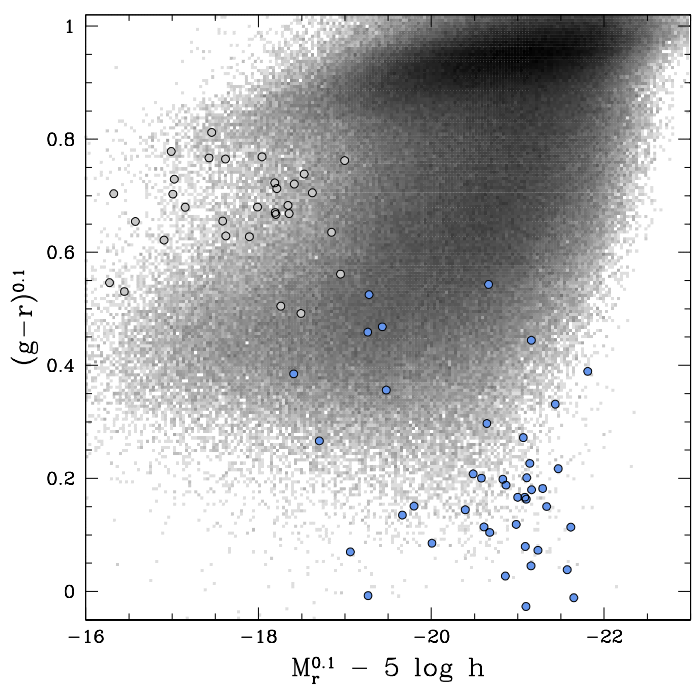

FIG. 9.- Mass-metallicity outliers on the color-magnitude diagram $\left[(g-r)\right.$ vs. $M_{r}$, both $k$-corrected to $z=0.1$ using the templates from Assef et al. (2008)] from the SDSS DR6 spectroscopic galaxy sample (Adelman-McCarthy et al. 2008). The blue circles denote the 42 low-metallicity high-mass galaxies in our sample; for reference, metal-rich dwarf galaxies from Paper I are denoted by the grey circles.

To seek to explain these galaxies' low oxygen abundances, we need to consider the population's other physical properties. As shown in Figure 9, the galaxies in our sample are all very blue; most - but not all - are also outliers in the galaxy color-magnitude diagram. In $\S 3.1$, we discuss how this blueness can be attributed to the galaxies' high specific star formation rates. Figure 2 shows that 40 out of 42 of the galaxies in our sample have disturbed morphologies suggestive of merging systems. Though some of the less-resolved galaxies in our sample may appear only slightly morphologically disturbed in the SDSS images, evidence suggests that, with higher resolution, these galaxies do in fact have unusual morphologies. In a Hubble Space Telescope study of local Lyman break galaxy (LBG) analogs, Overzier et al. (2008) found SDSSJ102613.97+4884458.9 $([\alpha, \delta]=[156.5580,48.7497]$, leftmost image on the bottom row of Figure 2) to have a strongly asymmetric profile with several distinct knots of star formation. In a preliminary study of the most UV luminous galaxies, Hoopes et al. (2007) found that these high specific star formation rate LBG analogs are metal-poor by $\sim 0.5$ dex relative to other galaxies of similar stellar mass. Hoopes et al. and Overzier et al. suggest that the observed high specific star formation rates and low metallicities for these LBG analogs are related to the galaxy collisions that formed these objects. (See also Struck et al. 2008 for a discussion of so-called "delayed" galaxies, i.e., high star formation rate interacting galaxies which have apparently managed to retain most of their gas until $z=0$.) In $\S 3.2$, we show how a simple merger-induced gas inflow picture can account for an offset in metallicity, and we discuss possible shortcomings in this interpretation. The other two galaxies in the sample are undisturbed spiral galaxies, and while these are clearly very interesting objects, we have no explanation to offer for their extreme offsets from the mass-metallicity relation. 


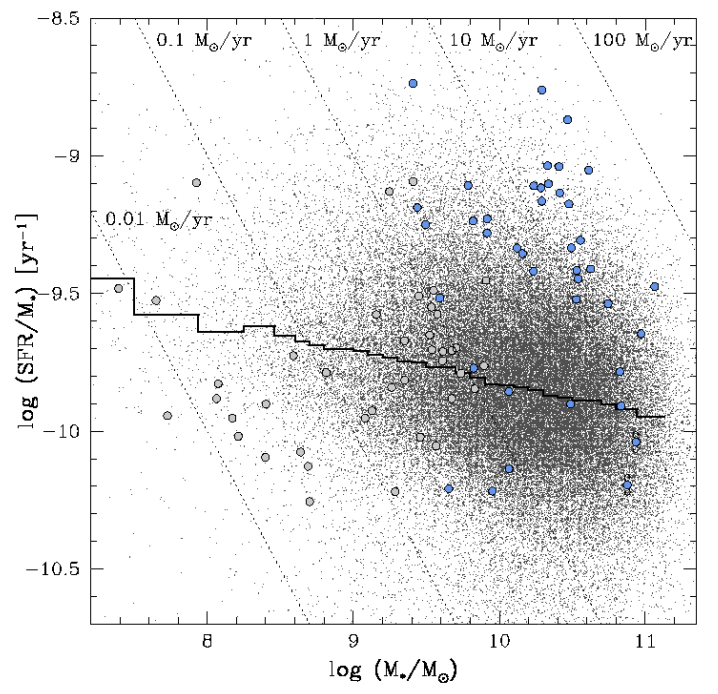

FIG. 10. - Specific star formation rate vs. stellar mass. The grey points are galaxies from the main Tremonti et al. (2004) SDSS sample described in $\S 2$, the low-metallicity massive galaxies in our sample are denoted with blue circles (with the only two clearly spiral galaxies marked by "§" symbols), and the metal-rich dwarf galaxies from Paper I are shown with large grey points for reference. The histogram denotes the median specific star formation rate as a function of stellar mass and the dotted lines show lines of constant star formation rate. The star formation rates estimates are from (Brinchmann et al. 2004) and the stellar mass estimates are from Tremonti et al. (2004) and Kauffmann et al. (2003); both have been aperture corrected.

\subsection{Star Formation Rates}

Ellison et al. (2008a) have shown that galaxies with higher specific star formation rates at a given mass have preferentially lower metallicities. In Figure 10, we plot the specific star formation rate (SSFR) as a function of stellar mass for the SDSS star-forming galaxies. Most (but not all) of our low-metallicity outliers clearly have higher specific star formation rates than typical of the larger sample. We plot the specific star formation rates against the mass-metallicity residual from Tremonti et al. (2004) in Figure 11; generally speaking, aperture corrections for the specific star formation rate only matter at the low SSFR end. While our lowmetallicity outliers do have higher-than-normal SSFRs, this relative difference alone is not enough to explain their low oxygen abundances.

\subsection{Observed Metallicity of Merging Galaxies}

As can be seen in Figure 2, most of galaxies in this sample are tidally interacting. Simulations have shown that galaxy major mergers are accompanied by starbursts and gas inflow from large galactocentric radii (e.g., Barnes \& Hernquist 1992; Mihos \& Hernquist 1996; Cox et al. 2006). Because typical spiral galaxies have metallicity gradients (Kennicutt et al. 2003), the inflowing gas will have a lower oxygen abundance than the native central gas. This suggests that perhaps our galaxies' observed low metallicities are a consequence of large quantities of low-metallicity gas from large galactocentric radii are being transported into the central few kiloparsecs and diluting the metal-rich central gas.

We can test this idea at the order of magnitude level to verify that it can give the observed oxygen abundance offsets of -0.3 to -0.8 dex (see, e.g., Fig. 7). The SDSS data measure the galaxies' central oxygen abundances; we wish to know what the change in oxygen abundance would be if low-metallicity gas from larger radii were dumped into the central regions. If we assume that all of the gas out to some large radius $R$ was to participate in this inflow, then the lower abundance would be the same as if we simply measured the total oxygen abundance for the entire galaxy gas disk out to the radius $R$. For reference, typical observed H I disks (e.g., Boomsma et al. 2008 in NGC 6946) have radii of $R \sim 20 \mathrm{kpc}$. We consider a model spiral galaxy with a gradient of $12+\log (\mathrm{O} / \mathrm{H})$ of slope $\Gamma_{\mathrm{O} / \mathrm{H}}$ in units of dex per kiloparsec; $\Gamma_{\mathrm{O} / \mathrm{H}}$ is and is typically of order $-0.05 \mathrm{dex} \mathrm{kpc}^{-1}$ (Zaritsky et al. 1994; van Zee et al. 1998; Kennicutt et al. 2003). We willl also assume that our model galaxy has a gas surface density $\Sigma$ that obeys a power-law relation with the radius such that $\Sigma(R)=\Sigma_{0}\left(R / R_{0}\right)^{\alpha}$. While a constant surface density $(\alpha=0)$ is a reasonable assumption at large radii (Wong \& Blitz 2002; Leroy et al. 2008), we will show that the calculated oxygen abundance dilution is not strongly dependent on the choice of this power-law slope. For notational simplicity, in this section only we will use $\mathrm{O} / \mathrm{H}$ as shorthand for the more cumbersome $12+\log (\mathrm{O} / \mathrm{H})$.

Let $\langle\mathrm{O} / \mathrm{H}\rangle_{\text {SDSS }}$ be the mean $\mathrm{O} / \mathrm{H}$ within an SDSS spectroscopic fiber of a spiral galaxy (i.e., within the central $3^{\prime \prime}$ diameter). Formally,

$$
\langle\mathrm{O} / \mathrm{H}\rangle_{\mathrm{SDSS}} \equiv \frac{\int_{0}^{R_{3}} \Sigma(R)(\mathrm{O} / \mathrm{H}) R \mathrm{~d} R \mathrm{~d} \theta}{\int_{0}^{R_{3}} \Sigma(R) R \mathrm{~d} R \mathrm{~d} \theta},
$$

where $R_{3}$ is the radius in kiloparsecs of the galaxy at a radius of $1.5^{\prime \prime}$ (i.e., spanned by the $3^{\prime \prime}$ SDSS fiber; this corresponds to $\approx 4.9 \mathrm{kpc}$ at $z=0.2$ ) and we are implicitly assuming the scale-height of the galaxy is roughly constant with radius. For radii $R>R_{3}$, the oxygen abundance is $\mathrm{O} / \mathrm{H}=(\mathrm{O} / \mathrm{H})_{0}+\Gamma_{\mathrm{O} / \mathrm{H}} R$, where $(\mathrm{O} / \mathrm{H})_{0}$ is the oxygen abundance extrapolated to $R=0$ using the adopted the abundance gradient. We can now expand Equation (5) to find

$$
\begin{aligned}
\langle\mathrm{O} / \mathrm{H}\rangle_{\mathrm{SDSS}} & =\left[\frac{2+\alpha}{2 \pi R_{3}^{2+\alpha}}\right] 2 \pi \int_{0}^{R_{3}} R^{\alpha}\left[(\mathrm{O} / \mathrm{H})_{0}+\Gamma_{\mathrm{O} / \mathrm{H}} R\right] R \mathrm{~d} R \\
& =(\mathrm{O} / \mathrm{H})_{0}+\left[\frac{2+\alpha}{3+\alpha}\right] \Gamma_{\mathrm{O} / \mathrm{H}} R_{3}
\end{aligned}
$$

Solving for the $R=0$ abundance, we find $(\mathrm{O} / \mathrm{H})_{0}=$ $\langle\mathrm{O} / \mathrm{H}\rangle_{\mathrm{SDSS}}-[(2+\alpha) /(3+\alpha)] \Gamma_{\mathrm{O} / \mathrm{H}} R_{3}$. The mean metallicity $\langle\mathrm{O} / \mathrm{H}\rangle_{\leq R}$ out to a radius $R$ is therefore

$$
\langle\mathrm{O} / \mathrm{H}\rangle_{\leq R}=\frac{\int_{0}^{R} \Sigma(R)(\mathrm{O} / \mathrm{H}) R \mathrm{~d} R \mathrm{~d} \theta}{\int_{0}^{R} \Sigma(R) R \mathrm{~d} R \mathrm{~d} \theta}=(\mathrm{O} / \mathrm{H})_{0}+\left[\frac{2+\alpha}{3+\alpha}\right] \Gamma_{\mathrm{O} / \mathrm{H}} R .
$$

Hence the change in metallicity $\Delta(\mathrm{O} / \mathrm{H})$ we would expect within an SDSS fiber diameter is $\Delta(\mathrm{O} / \mathrm{H})=[(2+\alpha) /(3+$ $\alpha)] \Gamma_{\mathrm{O} / \mathrm{H}} \Delta R=[(2+\alpha) /(3+\alpha)] \Gamma_{\mathrm{O} / \mathrm{H}}\left(R-R_{3}\right)$. For an outer radius of $R=20 \mathrm{kpc}$, an inner radius of $R_{3}=5 \mathrm{kpc}$, an abundance gradient of $\Gamma_{\mathrm{O} / \mathrm{H}}=-0.05 \mathrm{dex} \mathrm{kpc}^{-1}$, and 


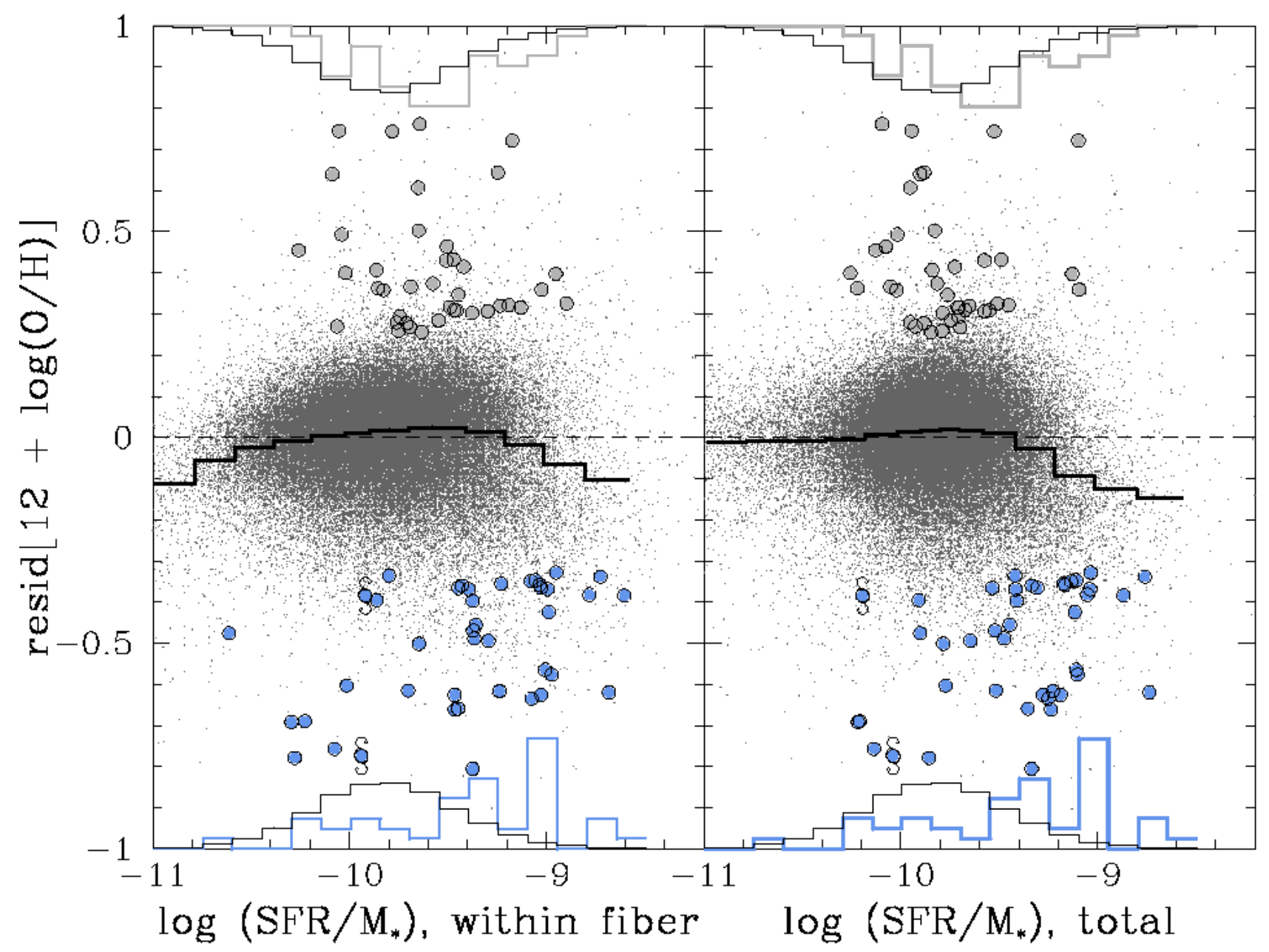

FIG. 11.- Residual in $12+\log (\mathrm{O} / \mathrm{H})$ from Tremonti et al. (2004) fit to the mass-metallicity relation v. specific star formation rate (SSFR), both within the SDSS $3^{\prime \prime}$ fiber (left) and aperture-corrected (right). In each panel, the blue points are the high-mass low-metallicity outliers in our sample while the large grey points are the metal-rich dwarf galaxies from Paper I. (The "§" symbols denote the only two clearly spiral galaxies in the low-metallicity high-mass sample.) The grey cloud of points are the galaxies from the main Tremonti et al. (2004) SDSS sample described in $\S 2$, and the central thick black histogram is the median residual $12+\log (\mathrm{O} / \mathrm{H})$ in bins of SSFR. The thin black histograms at the top and bottom of each panel are the distribution of SSFR of the parent sample, while the blue histograms at the bottom of each panel is the SSFR distribution of the low-metallicity outliers and the grey histogram at the top of each panel is the SSFR distribution of the high-metallicity outliers.

a flat radial gas-surface density distribution of $\alpha=0$, this leads to an oxygen abundance dilution of $\Delta(\mathrm{O} / \mathrm{H})=$ -0.5 dex, which is in the range we observe for these lowmetallicity outliers (see, e.g., Fig. 11).

We note that there are several caveats with this picture. First, we have assumed that the observed oxygen abundance corresponds to a mass-weighted abundance, whereas because we measure the metallicity via emission line spectra, it is actually weighted by the luminosities of the H II regions. Secondly, it is unclear if the timescales involved pose any problem. Simulations show mergers $d o$ induce gas inflow to the centers of galaxies (Cox et al. 2006), but most of this inflow occurs during the first close encounter between the two galaxies. While a few of the galaxies in our sample do have nearby neighbors, most do not: SDSS detects only a single, if disturbed, galaxy. It is possible that these are not yet dynamically relaxed; the two galaxies can pass close enough to one another that they are seen by SDSS as one object. If a merger-induced starburst lasts $\sim 1 \mathrm{Gyr}$ (Cox et al. 2006), then $\sim 1 \%$ of all (properly defined) starburst mergers should display lower-than-expected metallicities. The relevant dynamical timescale for gas inflow depends on the typical radius from which the gas originates. Because the dynamical time $t_{\text {dynam }} \approx R / v_{\text {esc }}$, gas at larger radii takes longer to reach $R_{3}$ and help dilute the central metallicity.

Several studies have shown that galaxies in close pairs tend to have lower metallicies than expected. Kewley et al. (2006a) found that galaxies separated by $\lesssim$ $20 h^{-1} \mathrm{kpc}$ have systematically lower oxygen abundances by $\sim 0.2$ dex. On the other hand, Ellison et al. (2008b) found an offset of $\sim 0.05 \mathrm{dex}$ in $12+\log (\mathrm{O} / \mathrm{H})$ at fixed mass galaxies in pairs with similar separations; the investigations of Michel-Dansac et al. (2008) of galaxies in close pairs suggest that the relative $\log (\mathrm{O} / \mathrm{H})$ offset is greater for the lower-mass galaxy in the pair than for the higher-mass galaxy. Kewley et al. also found relatively high specific star formation rates for the lowermetallicity member in their galaxy pairs; they attribute both the strong starburst activity and the lower oxygen abundance to fresh gas inflowing to the galaxies' central regions due to interaction with the closely neighboring 
galaxy.

In a similar vein, Lee et al. (2004) found that morphologically disturbed galaxies have systematically lower oxygen abundances (or higher luminosities) than galaxies of similar brightness (or metallicity). Likewise, luminous infrared galaxies (LIRGs), whose high specific star formation rates and highly disturbed morphologies are believed to be due to a recent major merger, are observed to have oxygen abundances that are $\sim 0.4$ dex lower than other galaxies of comparable masses (Rupke et al. 2008). The pronounced blue colors of our outliers (see Fig. 9) implies that while the star formation rates for many of these galaxies are consistent with those of LIRGs, these outliers are clearly not as dusty as their infrared-luminous cousins; it is unclear the extent to which these classes of galaxies are related, and why, if LIRGs do typically have such relatively low metallicities, no galaxies in our sample are extremely red.

\section{CONCLUCIONS AND IMPLICATIONS}

We have identified a sample of 42 low-metallicity highmass outliers from the mass-metallicity relation. As a population, these galaxies have disturbed morphologies and high specific star formation rates, implying that they are undergoing a merger-induced starburst. We propose that their observed low oxygen abundances are due the tidal interaction inducing large-scale gas inflow which subsequently dilutes the central interstellar medium in these galaxies. While there have been several observational and theoretical suggestions that interacting galaxies will result in a decreased observed gas-phase metallicity, this is the first work that has shown that the vast majority of severe low-metallicity outliers from the massmetallicity relation are morphologically disturbed. Finally, we note that the cuts outlined in $\S 2.1$ provide an effective means of using only colors and spectra to identify a rather pure (though not necessarily complete) sample of tidally disturbed galaxies.

One striking implication of these results is that, while it is safe to assume that the metallicities for populations of galaxies will fall within a particular range of values given their luminosities at redshifts for which the luminositymetallicity relation has been measured, one should not assign metallicities to individual galaxies based solely on their luminosities. For example, while studies of the host galaxies of long gamma-ray bursts (GRBs) suggest that GRBs are only found in low-metallicity environments (Stanek et al. 2006; Kewley et al. 2007), several recent GRBs have been associated with very luminous hosts, such as GRB 070306 and its $M_{B} \sim-22.3$ host galaxy (Jaunsen et al. 2008). As we have shown in this paper and Paper I, assuming an oxygen abundance for a galaxy from its luminosity alone can result in misestimating $12+\log (\mathrm{O} / \mathrm{H})$ in luminous galaxies by a whole dex, and thus one should not assume that, e.g., the host of GRB 070306 necessarily has a high metallicity. For cases in which $\log (\mathrm{O} / \mathrm{H})$ has been measured via emission line spectra for GRB hosts, it is generally low, even if the galaxy is luminous. For example, the host galaxy of GRB 031203 has an absolute $B$-band magnitude simi- lar to that of the Milky Way, yet Margutti et al. (2007) find it to have a low metallicity $(12+\log [\mathrm{O} / \mathrm{H}]=8.12)$ as well as a high star formation rate $\left(\sim 13 \mathrm{M}_{\odot} \mathrm{yr}^{-1}\right)$. Prochaska et al. (2004) interpret this offset in metallicity as a sign that GRB 031203 went off in a "very young star-forming region," which is in line with our interpretation in $\S 3.2$. Futhermore, the brighter GRB hosts studied by Fruchter et al. (2006) are morphologically very similar to our massive low-metallicity galaxies, and our results strongly suggest that the oxygen abundances inferred from luminosity alone are uncertain by as much as 1 dex. Specifically, if a luminous galaxy is morphologically disturbed, has a high specific star formation rate, and is extremely blue, then it should not be assumed to lie within the luminosity-metallicity locus of star-forming galaxies.

We would like to thank Roberto Assef for providing us with access to his low-resolution templates and codes for generating Figure 9 and Jason Eastman for assistance with making Figure 2. We are also grateful to David Weinberg for comments on the text as well as Todd Thompson, Paul Martini, and Scott Gaudi for helpful discussions. We further thank Sara Ellison, Maryam Modjaz, Janice Lee, Curt Struck, and Warren Brown for useful feedback on the first draft of this paper, as well as Roderik Overzier for pointing out to us that one of our outliers was in the Overzier et al. (2008) sample. Finally, we thank the anonymous referee for their thoughtful suggestions.

Funding for the SDSS and SDSS-II has been provided by the Alfred P. Sloan Foundation, the Participating Institutions, the National Science Foundation, the U.S. Department of Energy, the National Aeronautics and Space Administration, the Japanese Monbukagakusho, the Max Planck Society, and the Higher Education Funding Council for England. The SDSS Web Site is http://www.sdss.org/.

The SDSS is managed by the Astrophysical Research Consortium for the Participating Institutions. The Participating Institutions are the American Museum of Natural History, Astrophysical Institute Potsdam, University of Basel, University of Cambridge, Case Western Reserve University, University of Chicago, Drexel University, Fermilab, the Institute for Advanced Study, the Japan Participation Group, Johns Hopkins University, the Joint Institute for Nuclear Astrophysics, the Kavli Institute for Particle Astrophysics and Cosmology, the Korean Scientist Group, the Chinese Academy of Sciences (LAMOST), Los Alamos National Laboratory, the Max-Planck-Institute for Astronomy (MPIA), the MaxPlanck-Institute for Astrophysics (MPA), New Mexico State University, Ohio State University, University of Pittsburgh, University of Portsmouth, Princeton University, the United States Naval Observatory, and the University of Washington.

The STARLIGHT project is supported by the Brazilian agencies CNPq, CAPES and FAPESP and by the France-Brazil CAPES/Cofecub

\section{REFERENCES}

Adelman-McCarthy, J. K., et al. 2008, ApJS, 175, 297 Adelman-McCarthy, J. K., et al. 2006, ApJS, 162, 38
Assef, R. J., Kochanek, C. S., Brodwin, M., Brown, M. J. I., Caldwell, N., Cool, R. J., Eisenhardt, P., Eisenstein, D., Gonzalez, A. H., Jannuzi, B. T., Jones, C., McKenzie, E., Murray, S. S., \& Stern, D. 2008, ApJ, 676, 286 
Baldwin, J. A., Phillips, M. M., \& Terlevich, R. 1981, PASP, 93, 5 Barnes, J. E. \& Hernquist, L. 1992, ARA\&A, 30, 705

Boomsma, R., Oosterloo, T. A., Fraternali, F., van der Hulst, J. M., \& Sancisi, R. 2008, ArXiv e-prints, arXiv:0807.3339

Bresolin, F. 2006, ArXiv Astrophysics e-prints, astro-ph/0610690

Brinchmann, J., Charlot, S., White, S. D. M., Tremonti, C., Kauffmann, G., Heckman, T., \& Brinkmann, J. 2004, MNRAS, 351,1151

Cardelli, J. A., Clayton, G. C., \& Mathis, J. S. 1989, ApJ, 345, 245

Cid Fernandes, R., Mateus, A., Sodré, L., Stasińska, G., \& Gomes, J. M. 2005, MNRAS, 358, 363

Cox, T. J., Jonsson, P., Primack, J. R., \& Somerville, R. S. 2006, MNRAS, 373, 1013

Dalcanton, J. J. 2007, ApJ, 658, 941

Ellison, S. L., Patton, D. R., Simard, L., \& McConnachie, A. W. 2008a, ApJ, 672, L107

- 2008b, AJ, 135, 1877

Erb, D. K., Shapley, A. E., Pettini, M., Steidel, C. C., Reddy, N. A., \& Adelberger, K. L. 2006, ApJ, 644, 813

Finlator, K. \& Davé, R. 2008, MNRAS, 385, 2181

Fruchter, A. S., Levan, A. J., Strolger, L., Vreeswijk, P. M., Thorsett, S. E., Bersier, D., Burud, I., Castro Cerón, J. M., Castro-Tirado, A. J., Conselice, C., Dahlen, T., Ferguson, H. C., Fynbo, J. P. U., Garnavich, P. M., Gibbons, R. A., Gorosabel, J., Gull, T. R., Hjorth, J., Holland, S. T., Kouveliotou, C., Levay, Z., Livio, M., Metzger, M. R., Nugent, P. E., Petro, L., Pian, E., Rhoads, J. E., Riess, A. G., Sahu, K. C., Smette, A., Tanvir, N. R., Wijers, R. A. M. J., \& Woosley, S. E. 2006, Nature, 441, 463

Hoopes, C. G., Heckman, T. M., Salim, S., Seibert, M., Tremonti, C. A., Schiminovich, D., Rich, R. M., Martin, D. C., Charlot, S., Kauffmann, G., Forster, K., Friedman, P. G., Morrissey, P., Neff, S. G., Small, T., Wyder, T. K., Bianchi, L., Donas, J., Lee, Y.-W., Madore, B. F., Milliard, B., Szalay, A. S., Welsh, B. Y., \& Yi, S. K. 2007, ApJS, 173, 441

Jaunsen, A. O., Rol, E., Watson, D. J., Malesani, D., Fynbo, J. P. U., Milvang-Jensen, B., Hjorth, J., Vreeswijk, P. M., Ovaldsen, J.-E., Wiersema, K., Tanvir, N. R., Gorosabel, J., Levan, A. J., Schirmer, M., \& Castro-Tirado, A. J. 2008, ApJ, 681,453

Kauffmann, G., Heckman, T. M., White, S. D. M., Charlot, S., Tremonti, C., Brinchmann, J., Bruzual, G., Peng, E. W., Seibert, M., Bernardi, M., Blanton, M., Brinkmann, J., Castander, F., Csábai, I., Fukugita, M., Ivezic, Z., Munn, J. A., Nichol, R. C., Padmanabhan, N., Thakar, A. R., Weinberg, D. H., \& York, D. 2003, MNRAS, 341, 33

Kennicutt, Jr., R. C., Bresolin, F., \& Garnett, D. R. 2003, ApJ, 591,801

Kewley, L. J., Brown, W. R., Geller, M. J., Kenyon, S. J., \& Kurtz, M. J. 2007, AJ, 133, 882

Kewley, L. J. \& Dopita, M. A. 2002, ApJS, 142, 35

Kewley, L. J. \& Ellison, S. L. 2008, ApJ, 681, 1183

Kewley, L. J., Geller, M. J., \& Barton, E. J. 2006a, AJ, 131, 2004
Kewley, L. J., Groves, B., Kauffmann, G., \& Heckman, T. 2006b, MNRAS, 372, 961

Kobayashi, C., Springel, V., \& White, S. D. M. 2007, MNRAS, 376,1465

Larson, R. B. 1974, MNRAS, 169, 229

Lee, J. C., Salzer, J. J., \& Melbourne, J. 2004, ApJ, 616, 752

Leroy, A. K., Walter, F., Brinks, E., Bigiel, F., de Blok, W. J. G., Madore, B., \& Thornley, M. D. 2008, arXiv:0810.2556

Maiolino, R., Nagao, T., Grazian, A., Cocchia, F., Marconi, A., Mannucci, F., Cimatti, A., Pipino, A., Ballero, S., Calura, F., Chiappini, C., Fontana, A., Granato, G. L., Matteucci, F., Pastorini, G., Pentericci, L., Risaliti, G., Salvati, M., \& Silva, L. 2008, A\&A, 488, 463

Margutti, R., Chincarini, G., Covino, S., Tagliaferri, G., Campana, S., Della Valle, M., Filippenko, A. V., Fiore, F., Foley, R., Fugazza, D., Giommi, P., Malesani, D., Moretti, A., \& Stella, L. 2007, A\&A, 474, 815

McGaugh, S. S. 1991, ApJ, 380, 140

Michel-Dansac, L., Lambas, D. G., Alonso, M. S., \& Tissera, P. 2008, MNRAS, 386, L82

Mihos, J. C. \& Hernquist, L. 1996, ApJ, 464, 641

Overzier, R. A., Heckman, T. M., Kauffmann, G., Seibert, M., Rich, R. M., Basu-Zych, A., Lotz, J., Aloisi, A., Charlot, S., Hoopes, C., Martin, D. C., Schiminovich, D., \& Madore, B. 2008, ApJ, 677,37

Peeples, M. S., Pogge, R. W., \& Stanek, K. Z. 2008, ApJ, 685, 904

Pettini, M. \& Pagel, B. E. J. 2004, MNRAS, 348, L59

Prochaska, J. X., Bloom, J. S., Chen, H.-W., Hurley, K. C., Melbourne, J., Dressler, A., Graham, J. R., Osip, D. J., \& Vacca, W. D. 2004, ApJ, 611, 200

Rupke, D. S. N., Veilleux, S., \& Baker, A. J. 2008, ApJ, 674, 172

Savaglio, S., Glazebrook, K., Le Borgne, D., Juneau, S., Abraham, R. G., Chen, H.-W., Crampton, D., McCarthy, P. J., Carlberg, R. G., Marzke, R. O., Roth, K., Jørgensen, I., \& Murowinski, R. 2005, ApJ, 635, 260

Stanek, K. Z., Gnedin, O. Y., Beacom, J. F., Gould, A. P., Johnson, J. A., Kollmeier, J. A., Modjaz, M., Pinsonneault, M. H., Pogge, R., \& Weinberg, D. H. 2006, Acta Astronomica, 56, 333

Struck, C., Hancock, M., Smith, B. J., Appleton, P. N., Charmandaris, V., \& Giroux, M. 2008, in IAU Symposium, Vol. 244, IAU Symposium, ed. J. Davies \& M. Disney, 231-234

Tremonti, C. A., Heckman, T. M., Kauffmann, G., Brinchmann, J., Charlot, S., White, S. D. M., Seibert, M., Peng, E. W., Schlegel, D. J., Uomoto, A., Fukugita, M., \& Brinkmann, J. 2004, ApJ, 613,898

van Zee, L., Salzer, J. J., Haynes, M. P., O’Donoghue, A. A., \& Balonek, T. J. 1998, AJ, 116, 2805

Wong, T. \& Blitz, L. 2002, ApJ, 569, 157

Zaritsky, D., Kennicutt, Jr., R. C., \& Huchra, J. P. 1994, ApJ, 420, 87 